\title{
Case Report \\ Steroid sensitive recurrent painful ophthalmoplegic neuropathy: A case report
}

\author{
M Apirami ${ }^{1}$, T Kumanan ${ }^{2}$, K Sujanitha $^{2}$, M Malaravan $^{1}$ \\ ${ }^{1}$ Teaching Hospital, Jaffna, Sri Lanka, ${ }^{2}$ Faculty of Medicine, Jaffna
}

Keywords: recurrent painful ophthalmoplegic neuropathy, migraine, cranial nerve paresis, recurrent, oculomotor nerve thickening

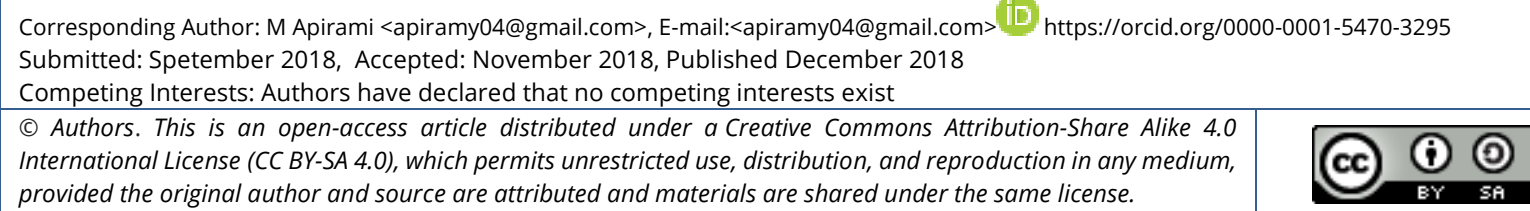

\section{Introduction}

Recurrent painful opthalmoplegic neuropathy (previously known as ophthalmic migraine) is a rare neurological syndrome characterized by recurrent episodes of isolated paresis or paralysis of the oculomotor nerve in a background of severe unilateral migranous headache [1]. It may be associated with paresis of the abducens and trochlear nerve.

There are two variants of the disease; childhood variant and adult variant. Most patients with the childhood variant have isolated third nerve involvement with evidence of MRI enhancement. Sixth and fourth nerve involvement is mostly seen in the adult variant but usually these patients have no MRI evidence. Oculomotor nerve paresis is noted in $83 \%$ of cases. These patients have pupillary dilatation and ptosis. Abducens nerve paresis is noted in $20 \%$ and trochlear nerve paresis in $2 \%$. Multiple paresis occurs in $6 \%$ of cases [2].

Opthalmoplegic neuropathy typically manifests in childhood. Aetio-pathogenesis is unclear, and may be ischaemic, inflammatory or demyelination. The patients experience a severe migranous headache [75\%] with MRI evidence of isolated oculomotor nerve enhancement, which suggests a migraine-related inflammatory role. Even though it has an excellent prognosis and usually results in complete recovery, some cases of permanent damage following frequent episodes of ocular neuropathy have been reported. Basic investigations, including inflammatory markers, virus study and CSF, are normal in recurrent painful opthalmoplegic neuropathy but MRI shows focal enhancement and thickening in $75 \%$ of the reported cases [2]. Corticosteroids are the mainstay of treatment. Although they may help in acute exacerbations, benefit in the progression of the disease is controversial.

Here we report a case of a young boy who presented with recurrent painful opthalmoplegic neuropathy without a past history of migrainous headache. Written informed consent was obtained from the patient for the publication of this report. 


\section{Case presentation}

An 18-year-old student presented with a history of left sided unilateral headache which lasted for a few hours with worsening severity. Later, he developed diplopia on horizontal and upward gaze which was accompanied with drooping of the left eye lid. He had no other focal neurological symptoms. He had had a similar episode a year previously, which had resolved completely within two weeks. He had undergone an otolaryngeal evaluation and neuroimaging during the previous episode and they were normal. He has a family history of migraine but has never had previous episodes of migraine. His ocular examination revealed 6/6 corrected vision in both eyes with normal anterior and posterior segments. On neurological examination, he had ophthalmoplegia and ptosis of the left eye, with a normal pupil and pupillary reflex (Figure 1).

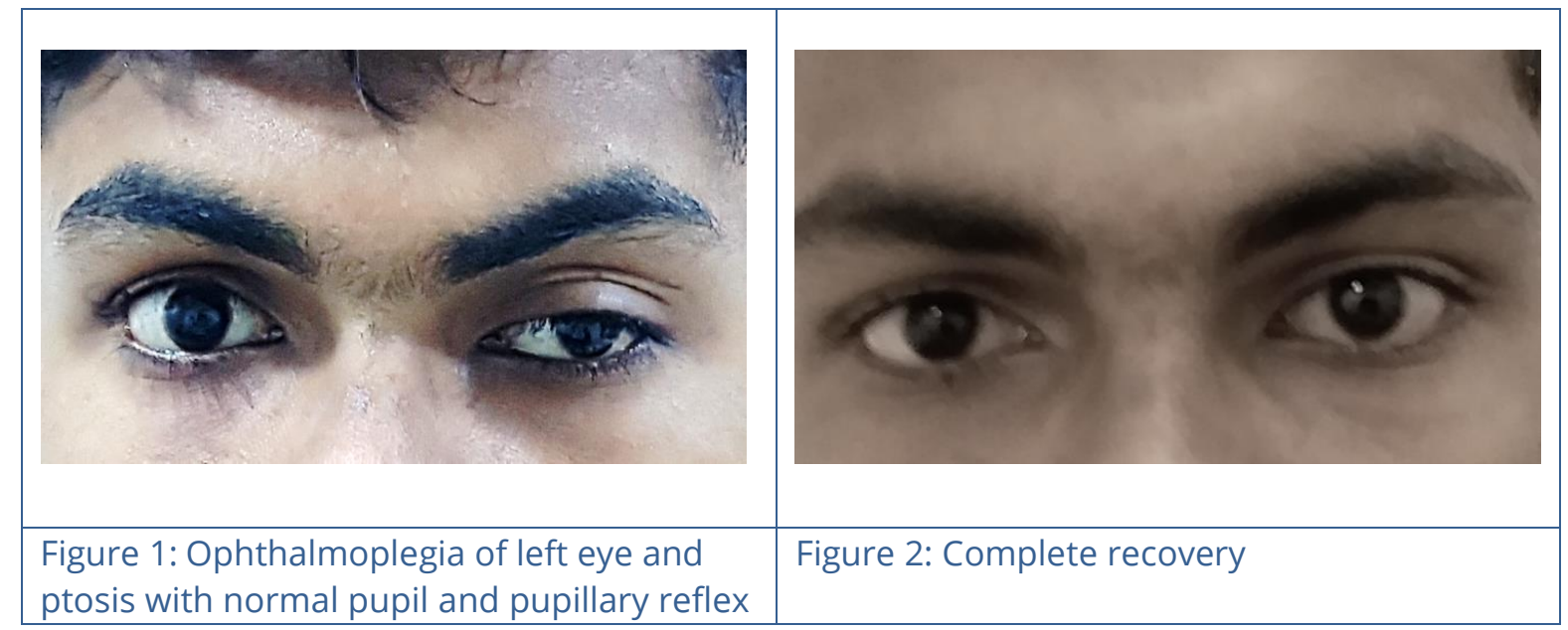

The rest of the physical examination was normal. A diagnosis of recurrent painful opthalmoplegic neuropathy [left partial 3rd nerve palsy with pupillary sparing] was made. He was started on steroids. After treatment he completely recovered, without any residual neurological deficits (Figure 2).

\section{Discussion}

Recurrent, painful opthalmoplegic neuropathy is a unique neurological syndrome of poorly known aetiology, with a prevalence as rare as 0.7 /million. [3] In 1980, it was thought to be a variant of migraine [1] and in 1988 it was classified as a subtype of migraine by the International Headache Society [1,3]. The second edition of the International Headache Society classifies this syndrome as cranial neuralgia $[1,2]$.

The pathogenesis was thought to be ischaemic neuropathy of the oculomotor nerve, caused by compression by the oedematous carotid artery inside the cavernous sinus, during a severe migrainous attack. But, in the imaging era, MRI has shown enhancement and thickening of the oculomotor nerve in three quarters of patients, particularly in the exit zone of midbrain. This has cast doubt on the theory of ischaemic neuropathy as it is not seen in other forms of ischaemic neuropathy such as diabetic neuropathy $[3,4]$ Later, it was thought to be post-viral or due to demyelination [3]. Normal CSF with negative viral studies and repeated involvement of a unilateral cranial nerve after complete recovery provides refutation for the above pathologies $[3,4]$ Migraine is said to have a definitive 
role in the pathogenesis of this neuropathy. This is evidenced by the fact that almost all patients develop ophthalmoplegia following a severe migraine attack, with ipsilateral involvement and normal CSF findings, and have a past history of typical migraine [5]. At present, it is thought that during a migraine attack the trigeminal vascular system is activated and neuropeptides are released in the vessel wall. These neuropeptides cause sterile inflammation of the vasa nervosa. This damages the blood nerve barrier and causes nerve injury and oedema. The MRI scan may or may not show enhancement [2]. When the migraine attack subsides, inflammation subsides and the oedema decreases. However, recurrent attacks may lead to permanent infarction.

Diagnosis of recurrent painful ophtalmoplegic neuropathy according to ICHD-3[6]

1) At least two attacks

2) Both of the following Unilateral headache Ipsilateral paresis of one, two or all 3 oculomotor nerves

3) Orbital, parasellar, or posterior fossa lesion has been excluded by appropriate investigation

4) NOT BETTER ACCOUNTED FOR BY ANOTHER ICHD-3 DIAGNOSIS

In summary, we report a case of recurrent painful opthalmoplegic neuropathy in an 18year-old boy who presented with oculomotor paresis without a history of previous episodes of migrainous headache. The paresis was recurrent, and he had complete recovery with the use of steroids [2].

\section{References}

1. Friedman DI. The ophthalmoplegic migraines: a proposed classification. https://doi.org/10.1111/j.1468-2982.2009.02004.x

2. Gelfand AA, Gelfand JM, Prabakhar P, Goadsby PJ. Ophthalmoplegic "migraine" or recurrent ophthalmoplegic cranial neuropathy: new cases and a systematic review. Journal of child neurology. 2012 Jun;27(6):759-66. https://doi.org/10.1177/0883073811426502

3. Lal V. Ophthalmoplegic migraine: Past, present and future. Neurology India. 2010 Jan 1;58(1):15. https://doi.org/10.4103/0028-3886.60388

4. Ravishankar K. Ophthalmoplegic migraine: still a diagnostic dilemma? Current pain and headache reports. 2008 Aug 1;12(4):285-91. https://doi.org/10.1007/s11916-008-0048-8

5. Ayele BA, Mengistu G, Wako AA. Adult Variant of Ophthalmologic Migraine with Recurrent 6th Cranial Nerve Palsy in 25yrs Old Ethiopian Patient: Case Report. J Neurol Stroke. 2016;4(6):00158.

6. Olesen J. Headache Classification Committee of the International Headache Society (IHS) The International Classification of Headache Disorders, Abstracts. Cephalalgia. 2018 Jan 1;38(1):1-211. https://doi.org/10.1111/j.1468-2982.2008.01709.x 